\title{
Solution Properties of Synthetic Polypeptides. XX. Light-Scattering Study of Poly-N5-(2-hydroxyethyl)L-glutamine in the Helix-Coil Transition Region
}

\author{
Tomohisa Ohta, ${ }^{*}$ Takashi Norisuye, Akio Teramoto, \\ and Hiroshi Fujita \\ Department of Polymer Science, Osaka University, \\ Toyonaka, Osaka, 560 Japan.
}

(Received November 20, 1975)

\begin{abstract}
The helix-coil transition of poly-N $\mathrm{N}^{5}$-(2-hydroxyethyl)L-glutamine (PHEG) in aqueous mixtures of 2-propanol was followed by means of light-scattering measurements. For each of the two samples investigated, the curve for the rootmean-square radius of gyration $\left\langle S^{2}\right\rangle^{1 / 2} \nu s$. helical fraction $f_{N}$ exhibited a broad minimum. This behavior was consistent with the previous optical rotatory dispersion data, which had indicated that the transition of PHEG was characterized by very low cooperativity. The data for $\left\langle S^{2}\right\rangle$ were analyzed by the method of Teramoto, et al., to give 14.7A for the effective bond length of the randomly coiled portions and $1.97 \mathrm{~A}$ for the pitch of the helix per monomer residue. The disparity of the latter value from the $1.5 \mathrm{~A}$ expected for the $\alpha$-helix was attributed to short-range interactions between the helical sections and the neighboring random coil residues. The Flory viscosity constant $\Phi$ was found to stay almost constant (about $2 \times 10^{21}$ ) over the range of $f_{N}$ up to 0.9 . This fact was taken to suggest that, for substantial portions of the transition, the average shape of a high molecular weight PHEG molecule is spherically symmetric like random coil.
\end{abstract}

KEY WORDS Polypeptide / Helix-Coil Transition / Light Scattering / Mean-Square Radius of Gyration / Poly- $\mathrm{N}^{5}$-(2-hydroxyethyl)L-Glutamine / Flory Viscosity Constant / $\alpha$-Helix /

In Part XVIII of this series of investigations, Miyake, et al., ${ }^{1}$ found from optical rotatory dispersion measurements that the cooperativity parameter $\sigma$ for the helix-coil transition of poly- $\mathrm{N}^{5}$-(2-hydroxyethyl)L-glutamine (PHEG) in aqueous mixtures of isopropanol was in the range between $1.5 \times 10^{-3}$ and $4.4 \times 10^{-3}$, depending on the 2-propanol content. The $\sigma$ values of this order of magnitude are much larger than $(0.06-3) \times 10^{-4}$ reported for other polypeptidesolvent systems, which implies that the transition of PHEG is much less cooperative. Miyake, et al., ${ }^{1}$ also found that the curve of intrinsic viscosity $[\eta] v s$. helical fraction $f_{N}$ for a highmolecular-weight sample of PHEG in aqueous 2-propanol had a broad minimum, and ascribed this behavior to the low cooperativity of the

* Present address: Hitachi Kasei Co., Ltd., Shimodate-shi, Ibaragi, Japan. transition. In this paper, the transition behavior of PHEG in aqueous 2-propanol is investigated by the light-scattering method. Data for the mean-square radius of gyration $\left\langle S^{2}\right\rangle$ are analyzed with the aid of the transition parameters determined by Miyake, et al., ${ }^{1}$ and the results are compared with those from our previous studies on poly- $\mathrm{N}^{5}$-(3-hydroxypropyl)L-glutamine (PHPG) in aqueous mixtures of methanol ${ }^{3}$ and $\operatorname{poly}(\gamma-$ benzyl L-glutamate) (PBLG) in dichloroacetic acid (DCA) containing $8.3 \mathrm{wt}-\%$ cyclohexanol, ${ }^{4}$ both of which undergo more cooperative transitions. Another topic in this paper is the relationship between $\left\langle S^{2}\right\rangle$ and $[\eta]$ for the interrupted helices of PHEG.

\section{EXPERIMENTAL}

Two fractionated samples of PHEG, A-I and A-II, were chosen from our stock. ${ }^{1}$ Light- 
scattering measurements were made at temperatures ranging from 5 to $40^{\circ} \mathrm{C}$ with water containing $0,25,40$, and $60 \mathrm{wt}-\%$ 2-propanol as solvent. Except for a few points, the same apparatus and experimental procedures as in our previous studies ${ }^{3,4}$ were employed. For the optical purification, solutions and solvents were spun at $2 \times 10^{4} \mathrm{~g}$ for $1-4 \mathrm{hr}$ in a Marusan centrifuge (Type 50V-S), followed by filtration through a Millipore filter (Type PHWP 04700). The Rayleigh ratio of benzene at $25^{\circ} \mathrm{C}$ was taken to be $46.5 \times 10^{-6}$ for the wavelength of $436 \mathrm{~nm} .^{5}$ The specific refractive index increments at $25^{\circ} \mathrm{C}$ were found to be $0.186_{5}\left(\mathrm{~cm}^{3} / \mathrm{g}\right)$ for pure water, $0.160_{9}$ for $25 \%$ 2-propanol, $0.157_{9}$ for $40 \%$ isopropanol, and $0.151_{0}$ for $60 \%$ 2-propanol, all at $436 \mathrm{~nm}$ wavelength. The viscosity measurements on sample A-II were made under solvent conditions similar to those for the light-scattering measurements.

\section{LIGHT-SCATTERING RESULTS}

Figures 1 and 2 show typical results from light-scattering measurements for sample A-I in $40 \%$ 2-propanol at $25^{\circ} \mathrm{C}$, where $K$ is the lightscattering constant, $R_{\theta}$ is the reduced intensity of scattered light at a scattering angle $\theta$, and $c$ is the concentration of the dissolved polymer in $\mathrm{g} / \mathrm{m} l$. Figure 3 illustrates plots of $\left(K c / R_{0}\right)^{1 / 2}$ $\left[=\left(K c / R_{\theta}\right)^{1 / 2}\right.$ at $\left.\theta=0\right] v s . c$ for sample A-II in mixtures of water and 2-propanol at $25^{\circ} \mathrm{C}$. The weight-average molecular weights $\bar{M}_{w}$ and the second virial coefficients $A_{2}$ obtained from the plots are summarized in Table I. The values of $\bar{M}_{w}$ for each sample derived at different solvent compositions agree with one another within experimental uncertainties, and those for sample A-I are close to $40.0 \times 10^{4}$ as determined previously by sedimentation equilibrium. ${ }^{1}$ The agreement of the $\bar{M}_{w}$ values determined in different solvents suggests that the effects of preferential adsorption on $\left\langle S^{2}\right\rangle$ and $A_{2}$ were negligible. It is to be noted that the values of $A_{2}$ for $60 \%$ 2-propanol solutions are vanishingly small, being of the order of magnitude observed for randomly coiled polymers in poor solvents. The data at other temperatures, though not shown here, displayed features similar to those described above.

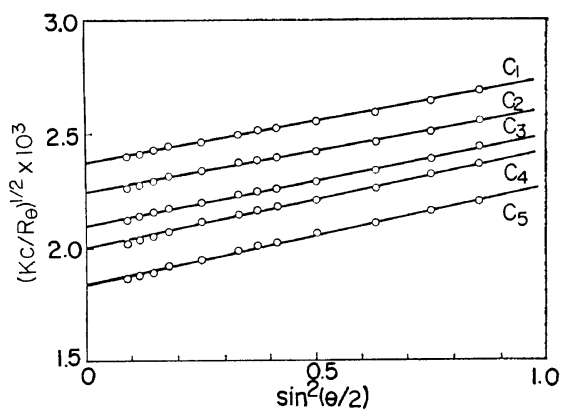

Figure 1. Plots of $\left(K c / R_{\theta}\right)^{1 / 2} v s . \sin ^{2}(\theta / 2)$ at fixed polymer concentration $c$ for PHEG A-I in $40 \mathrm{wt} \%$ 2-propanol at $25^{\circ} \mathrm{C}$. $c_{1}=0.4863 \times 10^{-2}, c_{2}=0.4044 \times$ $10^{-2}, \quad c_{3}=0.3267 \times 10^{-2}, \quad c_{4}=0.2712 \times 10^{-2}$, and $c_{5}=$ $0.1719 \times 10^{-2}(\mathrm{~g} / \mathrm{ml})$.

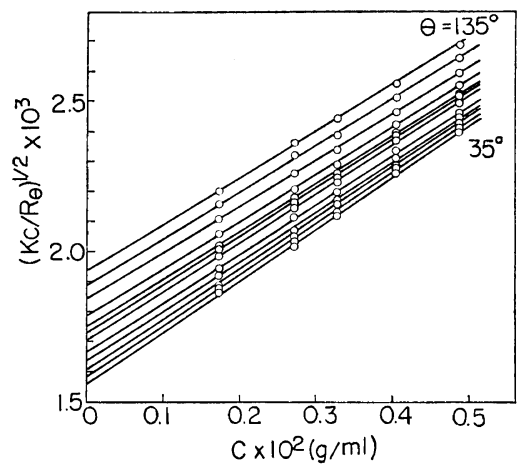

Figure 2. Plots of $\left(K c / R_{\theta}\right)^{1 / 2} v s . c$ at fixed scattering angle $\theta$ for PHEG A-I in 40\% 2-propanol at $25^{\circ} \mathrm{C}: \quad \theta=135,120,105,90,80,75,70,60,50,45$, 40 , and $35^{\circ}$ from top to bottom.

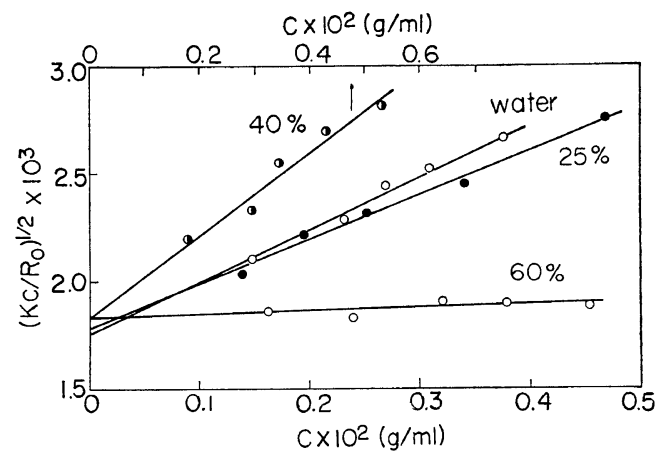

Figure 3. Plots of $\left(K c / R_{0}\right)^{1 / 2} v s . c$ for PHEG A-II in water-2-propanol mixtures of different compositions. 
Dimensional Change in the Helix-Coil Transition Region

Table I. Molecular weights and second virial coefficients for PHEG samples A-I and A-II

\begin{tabular}{|c|c|c|c|c|c|c|}
\hline $\begin{array}{l}\text { Sample } \\
\text { code }\end{array}$ & $\begin{array}{l}\text { Solvent composition, } \\
\text { wt- } \% \text { of } 2 \text {-propanol }\end{array}$ & $\bar{M}_{w} \times 10^{-4}$ & $\begin{array}{c}A_{2} \times 10^{4}, \\
\mathrm{~cm}^{3} \cdot \mathrm{mol} / \mathrm{g}^{2}\end{array}$ & $\bar{M}_{n} \times 10^{-4}$ & $\begin{array}{c}A_{2} \times 10^{4} \\
\mathrm{~cm}^{3} \cdot \mathrm{mol} / \mathrm{g}^{2}\end{array}$ & $\bar{M}_{w} / \bar{M}_{n}$ \\
\hline A-I & $\begin{array}{r}0 \\
25 \\
40 \\
60\end{array}$ & $\begin{array}{l}41.6 \\
40.0^{\mathrm{a}} \\
41.8 \\
41.3 \\
40.5\end{array}$ & $\begin{array}{l}3.29 \\
5.0^{\mathrm{a}} \\
3.39 \\
2.62 \\
0.90\end{array}$ & $30.9^{b}$ & $4.6^{\mathrm{b}}$ & $1.33^{\mathrm{d}}$ \\
\hline A-II & $\begin{array}{r}0 \\
25 \\
40 \\
60\end{array}$ & $\begin{array}{l}32.6 \\
31.8 \\
30.0 \\
30.0\end{array}$ & $\begin{array}{l}4.29 \\
3.74 \\
3.53 \\
0.36\end{array}$ & $26.0^{c}$ & $3.89^{c}$ & $1.20^{\mathrm{d}}$ \\
\hline
\end{tabular}

a Sedimentation equilibrium data taken from ref 1.

b Taken from ref 1 .

c Determined at $35^{\circ} \mathrm{C}$ on a Knauer membrane osmometer.

d Based on the averages of $\bar{M}_{w}$ determined under different experimental conditions.

Table II. Root-mean-square radii of gyration of PHEG samples A-I and A-II in water2-propanol mixtures

\begin{tabular}{|c|c|c|c|c|c|}
\hline \multirow{2}{*}{$\begin{array}{l}\text { Sample } \\
\text { code }\end{array}$} & \multirow{2}{*}{$\underset{{ }^{\circ} \mathrm{C}}{\text { Temp, }}$} & \multicolumn{4}{|c|}{$\left\langle S^{2}\right\rangle^{1 / 2}, \mathrm{~A}$} \\
\hline & & $0 \%$ & $\begin{array}{l}25 \% \\
\% \text { of }\end{array}$ & $\begin{array}{l}40 \% \\
\text { propa }\end{array}$ & $\begin{array}{l}60 \% \\
1)\end{array}$ \\
\hline \multirow[t]{8}{*}{ A-I } & 5 & 303 & 352 & 412 & 446 \\
\hline & 10 & - & 332 & 400 & 432 \\
\hline & 15 & 300 & 312 & 376 & 417 \\
\hline & 20 & - & 297 & 356 & 402 \\
\hline & 25 & 296 & 287 & 330 & 399 \\
\hline & 30 & - & 281 & 322 & 366 \\
\hline & 35 & 300 & 274 & 312 & 357 \\
\hline & 40 & - & 274 & 302 & 321 \\
\hline \multirow[t]{8}{*}{ A-II } & 5 & 253 & 292 & 346 & 365 \\
\hline & 10 & - & - & 323 & 338 \\
\hline & 15 & 248 & 258 & 303 & 330 \\
\hline & 20 & - & - & 271 & 311 \\
\hline & 25 & 231 & 238 & 251 & 307 \\
\hline & 30 & - & - & 242 & 290 \\
\hline & 35 & 238 & 231 & 229 & - \\
\hline & 40 & - & - & 226 & - \\
\hline
\end{tabular}

Figure 4 illustrates the angular dependence of the square root of the reciprocal particle scattering function $P(\theta)^{-1}$ for sample A-I in $60 \%$ and 25\% 2-propanol. In Figure 5 is depicted the temperature dependence of $\left\langle S^{2}\right\rangle^{1 / 2}$ for sample A-I in water-2-propanol mixtures of varying compositions. With the data of the Moffitt parameter $b_{0}$ reported for the same system in Part XVIII (see Figure 3 of ref 1 ), the observed variations of $\left\langle S^{2}\right\rangle^{1 / 2}$ with either temperature or solvent composition may be taken as a

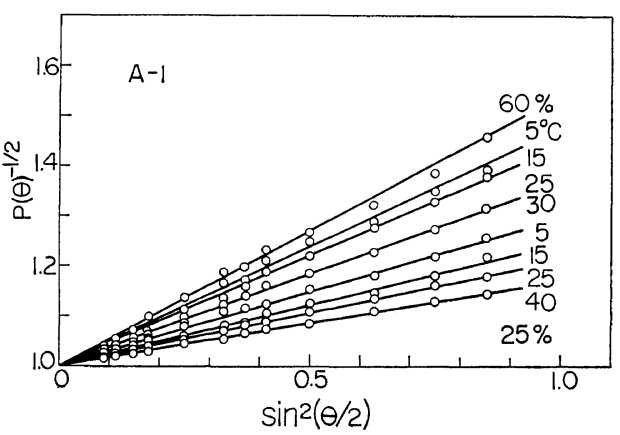

Figure 4. Inverse square root of particle scattering function $P(\theta)$ plotted against $\sin ^{2}(\theta / 2)$ for PHEG A-I in $60 \%$ and $25 \%$ 2-propanol at the indicated temperatures.

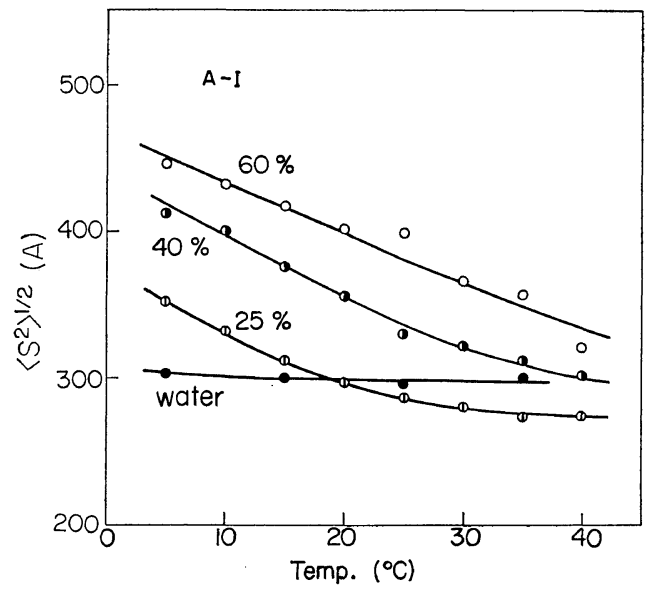

Figure 5. Temperature dependence of $\left\langle S^{2}\right\rangle^{1 / 2}$ for sample A-I in aqueous 2-propanol of the indicated compositions. 


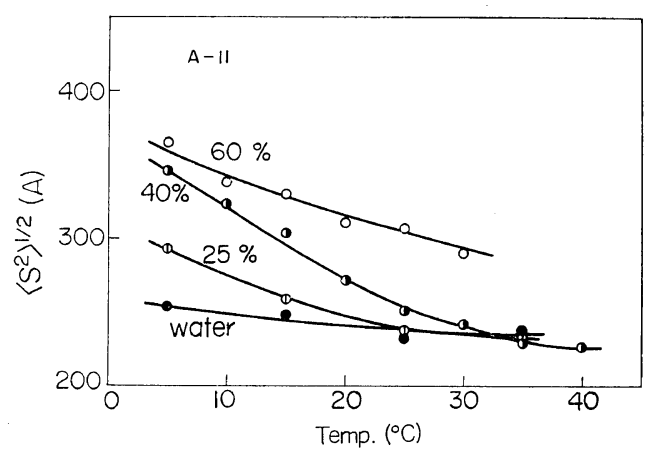

Figure 6. Temperature dependence of $\left\langle S^{2}\right\rangle^{1 / 2}$ for sample A-II in aqueous 2-propanol.

manifestation of the coil-to-helix transition of the PHEG molecule. Figure 6 shows the data for sample A-II, which have features essentially similar to those for sample A-I.

\section{DISCUSSION}

Behavior of $\left\langle S^{2}\right\rangle$ in the Helix-Coil Transition Region

The data of $f_{N}$ obtained for PHEG in Part $\mathrm{XVIII}^{1}$ can be utilized to see the change in $\left\langle S^{2}\right\rangle$ with the helical fraction. Figure 7 displays such changes for sample A-II at different solvent compositions. It is seen that the data points for different compositions approximately form a single curve. As $f_{N}$ increases, the curve initially declines, passes through a broad minimum, and then rises sharply. Similar data for sample A-I are shown in Figure 8, in which the dashed line represents the relation between $\left\langle S^{2}\right\rangle^{1 / 2}$ and $f_{N}$ established previously ${ }^{4}$ for a PBLG sample with nearly the same degree of polymerization (weight-average degree of polymerization $\bar{N}_{w}=2200$ ) in DCA containing 8.3 wt- $\%$ cyclohexanol. The curves for PHEG and PBLG give nearly the same values for the $\left\langle S^{2}\right\rangle^{1 / 2}$ at the limit of random coil, i.e., $f_{N}=0$, but follow markedly different paths in the intermediate region of $f_{N}$. This difference may primarily be attributed to a large difference in the cooperativity parameter between PBLG in the DCA mixture $\left(0.85 \times 10^{-4}\right)^{4}$ and PHEG in aqueous isopropanol $\left[(1.5-4.4) \times 10^{-3}\right] .^{1} \quad$ The theory ${ }^{6,7}$ predicts that, when compared at the same value of $f_{N},\left\langle S^{2}\right\rangle$ is smaller for larger $\sigma$.

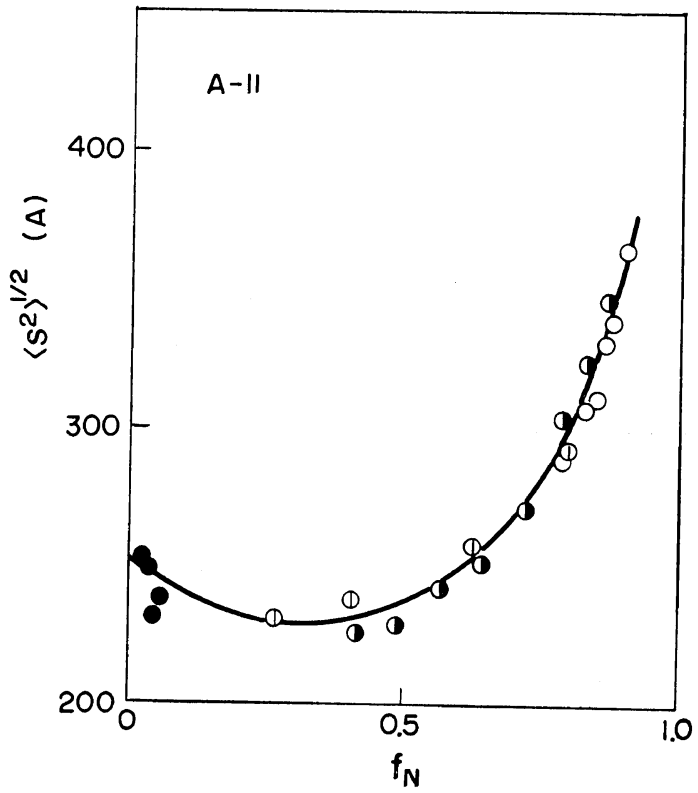

Figure 7. Plots of $\left\langle S^{2}\right\rangle^{1 / 2} v s$. helical content $f_{N}$ for sample A-II in aqueous 2-propanol. The symbols are the same as in Figure 5. The values for $f_{N}$ were taken from ref 1 .

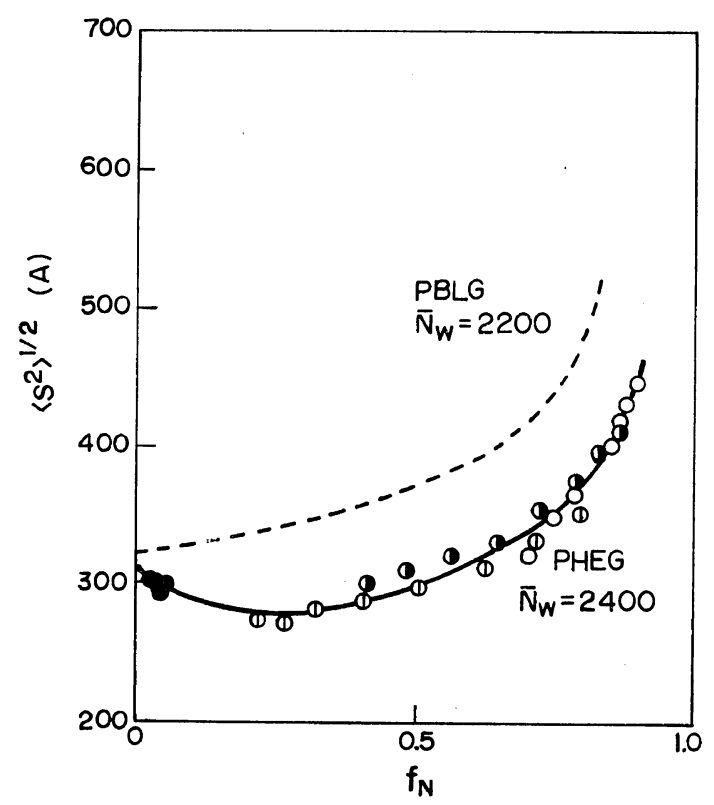

Figure 8. Plots of $\left\langle S^{2}\right\rangle^{1 / 2} v s . f_{N}$ for PHEG A-I in aqueous 2-propanol. The symbols are the same as in Figure 5. The dashed line refers to PBLG $\left(\bar{N}_{w}=2200\right)$ in dichloroacetic acid contaning 8.3 wt\% cyclohexanol. ${ }^{4}$ 
Analysis of $\left\langle S^{2}\right\rangle$ Data by Nagai's Theory

In Part VII, ${ }^{8}$ a simple approximate expression for $\left\langle S^{2}\right\rangle$ of interrupted helices was derived from Nagai's exact one ${ }^{6}$ under the conditions that $\sigma^{1 / 2} \ll 1, N \gg 1$, and $N \sigma^{1 / 2}>2$; this was utilized to work out a method which allows the size parameters $a_{0}$ and $a_{1}$ of the chain to be determined from experiment. Here $N$ is the degree of polymerization, $a_{0}$ is the effective bond length of the randomly coiled portions in an interrupted helical chain, and $a_{1}$ is the pitch per monomeric residue in its helical portions. The method has been applied successfully to $\mathrm{PHPG}^{3}$ and PBLG. ${ }^{4}$ However, since the values of $\sigma^{1 / 2}$ for PHEG in aqueous isopropanol are not always much smaller than unity, the present $\left\langle S^{2}\right\rangle$ data cannot be applied to the method. Therefore we here start with Nagai's exact expression for $\left\langle S^{2}\right\rangle$, which can be recast in the form.

with

$$
\dot{H}_{1} / H_{2}=a_{0}^{2}+a_{1}^{2} H_{3} / H_{2}
$$

$$
H_{1}=6\left\langle S^{2}\right\rangle / N(1-f)
$$

where $f$ is the value of $f_{N}$ at infinitely large $N$ and $\mathrm{H}_{2}$ and $\mathrm{H}_{3}$ are complicated functions of $N, \sigma$, and $f$. Equation 1, which has the same form as the approximate expression of Teramoto, et al., ${ }^{8}$ predicts that if both $a_{0}$ and $a_{1}$ are constant over a substantial range of a transition from random coil to helix, a plot of $H_{1} / H_{2} v s$. $H_{3} / H_{2}$ (referred to as the $H$-plot) should give a straight line, regardless of solvent conditions and the molecular weight of the sample. Then $a_{0}$ and $a_{1}$ may be evaluated from the intercept and slope of the straight line, respectively.

We computed $H_{1} / H_{2}$ and $H_{3} / H_{2}$ by substituting into Nagai's exact expression (eq 97-102 of ref 6) the present data for $\left\langle S^{2}\right\rangle$ and $\bar{N}_{w}$ together with the values of $\sigma$ and $f$ determined in Part XVIII. ${ }^{1}$ The resulting $H$-plot is shown in Figure 9, in which the insert gives an enlarged plot for smaller values of $\mathrm{H}_{3} / \mathrm{H}_{2}$. The plotted points

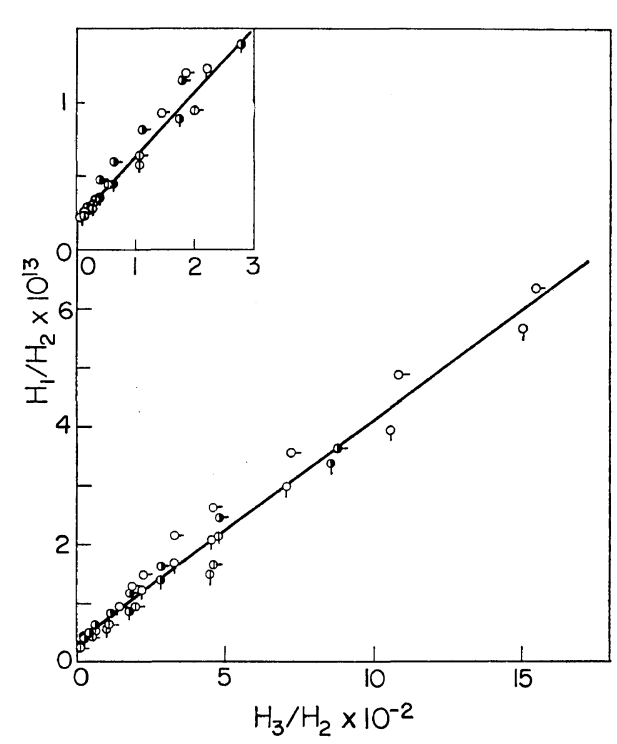

Figure 9. Analysis of $\left\langle S^{2}\right\rangle$ data for PHEG in aqueous 2-propanol in terms of $H$-plot. The horizontal and vertical pips refer to A-I and AII, respectively, and the other symbols are the same as in Figure 5.

scatter around a straight line, which, according to eq 1 , yields $a_{0}=14.7 \mathrm{~A}$ and $a_{1}=1.97 \mathrm{~A}$. These values are compared with those for $\mathrm{PHPG}^{3}$ and $\mathrm{PBLG}^{4}$ in Table III. The $a_{0}$ value of $14.7 \mathrm{~A}$ for PHEG is quite close to $15.0 \mathrm{~A}$ for PHPG, but much larger than 11.3A deduced by Brant and Flory $^{9}$ for unperturbed polypeptide chains. This disparity may be attributable to excludedvolume effects, as has been advocated previously. $^{3,4}$ The $a_{1}$ value of $1.97 \mathrm{~A}$ for PHEG is appreciably larger than those for PHPG and PBLG, which happen to be close to $1.5 \mathrm{~A}$ expected for the $\alpha$-helix. From the reported ORD and $C D$ data, ${ }^{1,10}$ the possibility of a different helical conformation than the $\alpha$-helix must be rejected for PHEG. The effect of the polydispersity of the samples must have been small when judged from the ratios of $\bar{M}_{w}$ to $\bar{M}_{n}$ given

Table III. Size parameters $a_{0}$ and $a_{1}$ for interrupted helices of polypeptides

\begin{tabular}{cccccl}
\hline Polypeptide & Solvent & $a_{0}, \mathrm{~A}$ & $a_{1}, \mathrm{~A}$ & $\sigma \times 10^{4}$ & \multicolumn{1}{c}{ Reference } \\
\hline PHEG & water-2-propanol & 14.7 & 1.97 & $15-44$ & This work \\
PHPG & water-methanol & 15.0 & 1.65 & 3.2 & Okita, et al. ${ }^{3}$ \\
PBLG & DCA-cyclohexanol & 16.9 & 1.53 & 0.85 & Norisuye, et al. $^{4}$ \\
Unperturbed chain & - & 11.3 & - & - & Brant and Flory \\
\hline
\end{tabular}


in Table I. It is also difficult to attribute the disparity to the excluded-volume effects, because the values of $A_{2}$ for $60 \%$ 2-propanol solutions are quite small.

To obtain a more detailed insight into the conformation of polypeptides with very large $\sigma$, such as PHEG, we computed the average number $g_{N}$ of helical sections in a PHEG molecule of $N=2400$ (corresponding to sample A-I) by means of the approximate expression of Teramoto, et $a l .,{ }^{8}$ and with use of the data for $s$ and $\sigma$ given earlier. ${ }^{1}$ The results were that $g_{N}=46$ for $25 \%$ 2-propanol, 58 for 40\% 2-propanol, and 56 for $60 \%$ 2-propanol, all at $25^{\circ} \mathrm{C}$. These $g_{N}$ values give for the average lengths of the helical sections in the respective solvent mixtures at $25^{\circ} \mathrm{C}$ the values of 32,40 , and $54 \mathrm{~A}$, when the experimental values of $f_{N}$ are inserted and the helix pitch per monomer unit is taken to be 1.5A. Since the effective bond length $a_{0}$ of the randomly coiled portions is 11-17A (see Table III), the average length of one helical section is only $2-5$ times as large as $a_{0}$ for all the mixed solvents studied. This fact implies that the conformation of the PHEG molecule is not very much different from random coils even when the value of $f_{N}$ increases up to 0.83 in $60 \%$ isopropanol at $25^{\circ} \mathrm{C}$. The Nagai theory takes no account of short-range interactions between helical sections and their adjacent randomly coiled residues. Hence the helix pitch $a_{1}$ evaluated by this theory should be regarded as an effective one, as should be the case with $a_{0}$. A more reasonable analysis of the present data may be made by resort to the theory of Miller and Flory, which allows for such interferences. This task is left for our future work.

Relationship between $\left\langle S^{2}\right\rangle$ and $[\eta]$

Figure 10 shows the variation of intrinsic viscosity [ $\eta$ ] with $f_{N}$ for sample A-II in aqueous 2-propanol. The features of the curve are quite similar to those reported previously ${ }^{1}$ for sample A-I in the same mixed solvents. Comparison of Figure 10 with Figure 7 reveals the existence of a close relation between $[\eta]$ and $\left\langle S^{2}\right\rangle$ for the PHEG molecule. The relation may be examined by applying the Flory-Fox empirical equation ${ }^{11}$

$$
[\eta]=\Phi\left(6\left\langle S^{2}\right\rangle\right)^{3 / 2} / \bar{M}_{w}
$$

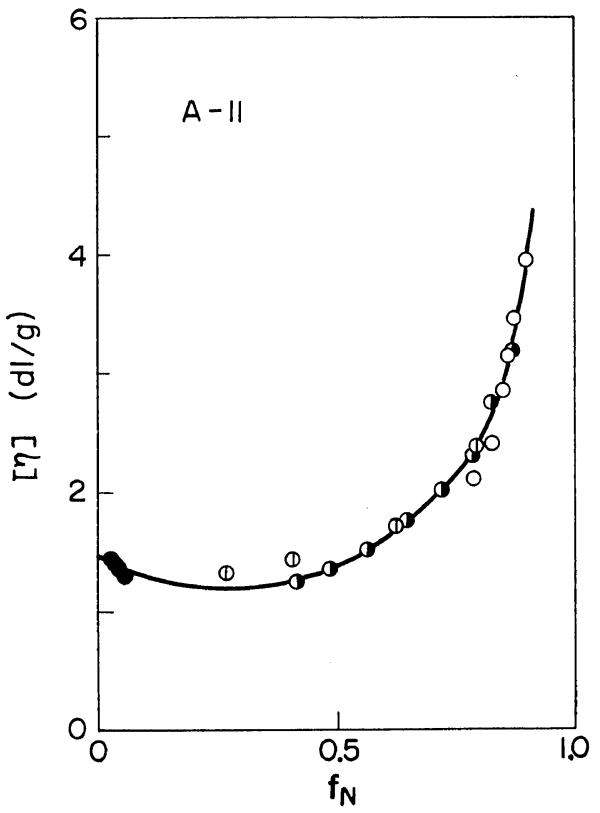

Figure 10. Plots of $[\eta] v s . f_{N}$ for PHEG A-II in aqueous 2-propanol. The symbols are the same as in Figure 5.

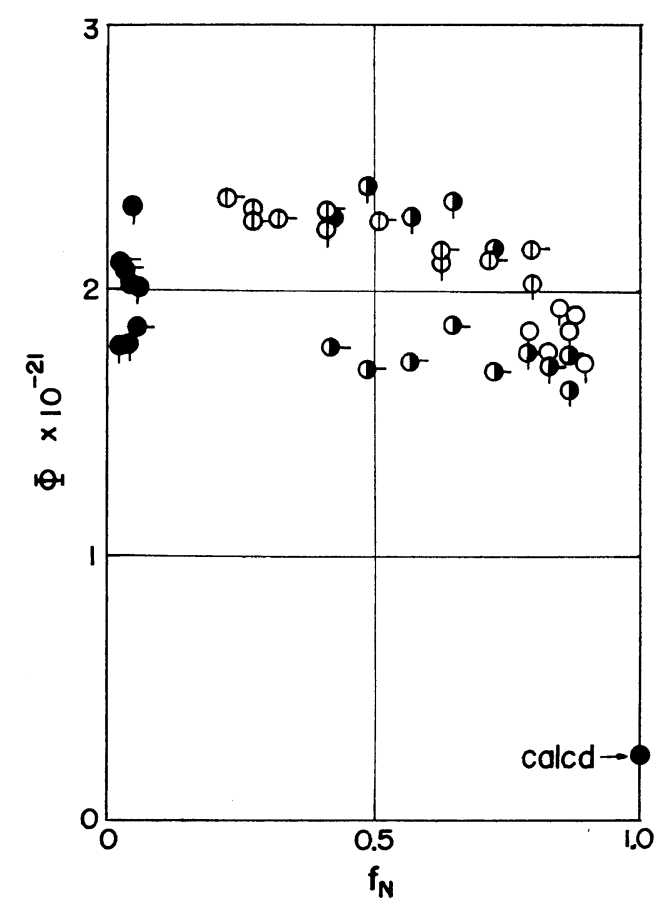

Figure 11. Flory viscosity constant $\Phi$ plotted against $f_{N}$ for PHEG in aqueous 2-propanol. The symbols are the same as in Figure 9. For the value of $\Phi$ at $f_{N}=1$, see the text. 
where $\Phi$ is the Flory viscosity constant.

Figure 11 shows $\Phi$ values plotted against $f_{N}$. For the sake of reference, we have plotted at $f_{N}=1$, the value of $\Phi$ calculated from $[\eta]=8.9$ $\mathrm{d} l / \mathrm{g}$ for sample A-I in the helicogenic solvent trifluoroethanol at $25^{\circ} \mathrm{C}$, and the value of $\left\langle S^{2}\right\rangle$ for the rigid $\alpha$-helix of $N=2400$. All the $\Phi$ values stay almost constant (about $2 \times 10^{21}$ ), the known average for randomly coiled polymers in good solvents. These results suggest that the conformation of PHEG in aqueous 2-propanol is not very much different from a random coil over a substantial region of the helix-coil transition. The present results differ from Norisuye's for PBLG in DCA containing 8.3 wt- $\%$ cyclohexanol, ${ }^{2,12}$ in which $\Phi$ were constant (about $1.9 \times 10^{21}$ ) for $f$ lower than 0.6 but decreased sharply as $f$ approached unity. The difference between the two systems again may be attributed to their appreciably different cooperativities.

Acknowledgments. The authors wish to thank Prof. P. J. Flory for his kind comments concerning the theoretical aspects of this work.

\section{REFERENCES}

1. M. Miyake, S. Akita, A. Teramoto, T. Norisuye, and H. Fujita, Biopolymers, 13, 1173 (1974).

2. A. Teramoto and H. Fujita, Advan. Polym. Sci., 18, 65 (1975).

3. K. Okita, A. Teramoto, and H. Fujita, Polymer J., 1, 582 (1970).

4. T. Norisuye, A. Teramoto, and H. Fujita, ibid., 4, 323 (1973).

5. Gj. Dezělić and J. Vavra, Croat. Chem. Acta, 38, 35 (1966).

6. K. Nagai, J. Chem. Phys., 34, 887 (1961).

7. W. G. Miller and P. J. Flory, J. Mol. Biol., 15, 298 (1966).

8. A. Teramoto, T. Norisuye, and H. Fujita, Polymer J., 1, 55 (1970).

9. D. A. Brant and P. J. Flory, J. Amer. Chem. Soc., 87, 2788 (1965).

10. N. Lotan, A. Yaron, and A. Berger, Biopolymers, 4, 368 (1966).

11. P. J. Flory and T. G. Fox, J. Amer. Chem. Soc., 73, 1904 (1951).

12. T. Norisuye, Thesis, Osaka University, 1973. 